\title{
Acceptance and Commitment Based Therapy on Disease Perception and Psychological Capital in Patients with Type II Diabetes
}

\section{ARTICLE INFO}

\section{Article Type}

Original Research

\section{Authors}

Baghban Baghestan A. ${ }^{1} M A$

Aerab Sheibani Kh.* $P h D$

Javedani Masrur M. ${ }^{2} P h D$

How to cite this article
Baghban Baghestan A, Aerab Sh-
eibani Kh, Javedani Masrur M.
Acceptance and Commitment
Based Therapy on Disease Per-
ception and Psychological Capi-
tal in Patients with Type II
Diabetes. Horizon of Medical Sc-
iences. 2017;23(2):135-140.

*Psychology Department, Humanities Faculty, Payam-e-Noor University, Tehran, Iran

${ }^{1}$ Psychology Department, Humanities Faculty, Payam-e-Noor University, Tehran, Iran

${ }^{2}$ Obstetrics \& Gynecology Department, Medicine Faculty, Iran University of Medical Sciences, Tehran, Iran

\section{Correspondence}

Address: Humanities Faculty, Payam-E-Noor University, Alghadir Square, Ferdows City, South Khorasan, Iran. Postal Code: 9771853144

Phone: +98 (56) 32721800

Fax: +98 (56) 32727999

shakiba_a_shaibani@yahoo.com

\section{Article History}

Received: May 19, 2016

Accepted: December 20, 2016

ePublished: March 25, 2017

\section{A B S T R A C T}

Aims As a prevalent metabolic disease, diabetes can be followed by severe mental outcomes leading to problems affecting the daily life. The aim of the study was to determine the effects of acceptance and commitment-based intervention on illness perception and psychological capital in persons with type II diabetes.

Materials \& Methods In the controlled pretest-posttest semi-experimental study, 34 patients with type II diabetes were studied in the Diabetes Clinic of Chamran Hospital of Ferdows City in 2015. The subjects, selected via available sampling method, were randomly divided into two groups including control ( $\mathrm{n}=17$ persons) and experimental $(\mathrm{n}=17$ persons) groups. Data was collected by short illness perception questionnaire (IPQ) and Luthans' psychological capital questionnaire (PCQ). Eight 60-minute acceptance and commitmentbase intervention sessions were weekly conducted in experimental group. Data was analyzed by SPSS 18 software using descriptive statistics and covariance analysis test.

Findings The pretest score having been adjusted, the acceptance and commitment-based intervention significantly increases the scores of illness perception and its sub-scales $(p=0.0001)$ except the personal control sub-scale. In addition, it significantly increases the scores of the psychological capital and its sub-scales $(p=0.0001)$ in patients with type II diabetes.

Conclusion The acceptance and commitment-based intervention can considerably improve the illness perception and the psychological capital in persons with type II diabetes.

Keywords Intervention; Acceptance and Commitment Therapy; Diabetes Melitus, Type II

\section{CIT A T I O N L I N KS}

[1] Promotion of psychological capital model in diabetic pation ... [2] Costeffectivenessof a stepped-care intervention to prevent ... [3] Comparing effects of walking and yoga exercise on quality ... [4] Role of yoga in preventing and controlling type 2 diabetes ... [5] Prevalence of type 2 diabetes mellitus in Iran and its relationship with gender, urbanisation, education ... [6] The influence of yoga-based programs on risk profiles in adults ... [7] Health related quality of life in ... [8] The present status of diabetes education and the role recognition as a diabetes ... [9] The Relationship between illness perception and ... [10] Illness perception inpatients ... [11] A review of psychological correlates of adjustment ... [12] The mediating effects of coping strategies in the relationship ... [13] Patient illness perceptions and coping ... [14] The need for and meaning of positive ... [15] The world of ... [16] Relationship of core self-evaluations to goal ... [17] Relationship of core self-evaluations traits -self-esteem ... [18] Relationships between psychological ... [19] Improving mental health status and coping abilities ... [20] Organisational ... [21] Sleep deprivation reduces perceived emotional ... [22] The effectiveness of acceptance and ... [23] Acceptance and commitment therapy ... [24] Meta-analysis of psychological interventions for chronic ... [25] Systematic review and meta ... [26] The role of mindfulness and psychological ... [27] Acceptance and values-based action ... [28] Can exposure strategies ... [29] "Giving It Space" a case study ... [30] The effectiveness of an acceptance and commitment ... [31] The effectiveness of acceptance and commitment [32] Quantitative and qualitative research methods ... [33] The brief illness perception ... [34] The Moderating Role of Coping Strategies in Relationship ... [35] Positive psychological capital: Measurement and relationship ... [36] Role of psychological capital in psychological wellbeing by considering ... [37] Acceptance and commitment ... [38] The effects of acceptance versus control ... [39] Guerrero LF. DBT, FAP and ACT: How empirically oriented are the new behavior ... 
از سوى ديخر، شناخت بيماران از بيماريشان تحت عنوان ادراك باري

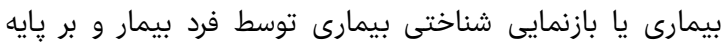

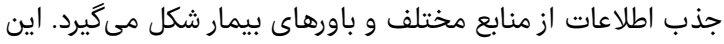

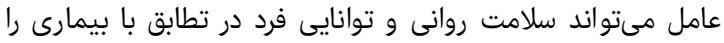

$$
\text { تحت تاثير قرار دهد [9]. }
$$

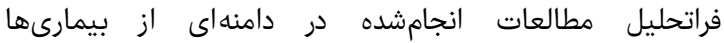

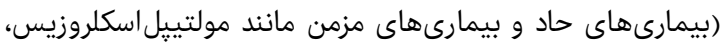

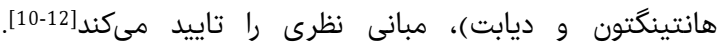

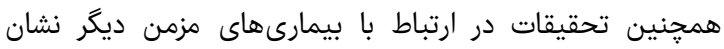

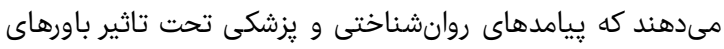

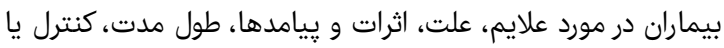

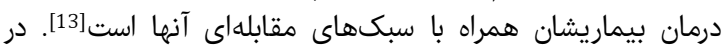

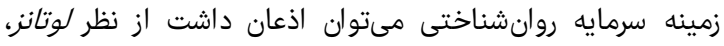

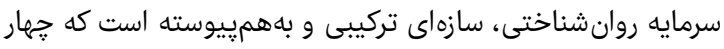

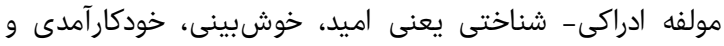

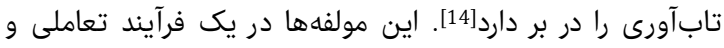

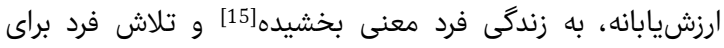

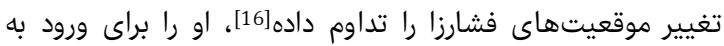

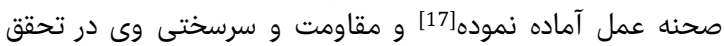

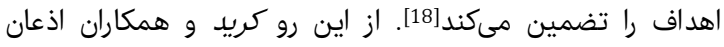

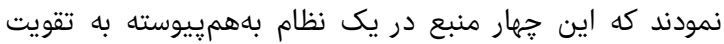

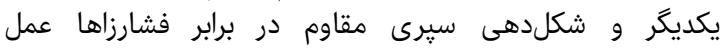

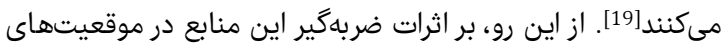

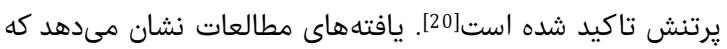

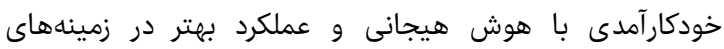

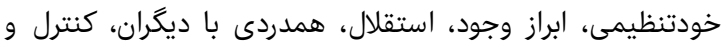
خوشبينى رابطه دارد[15-21].

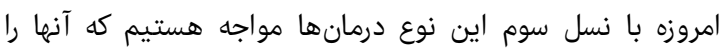

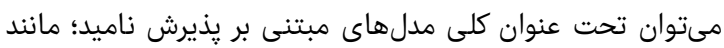

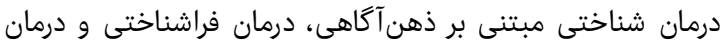

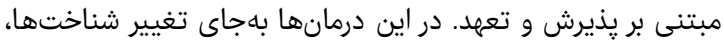

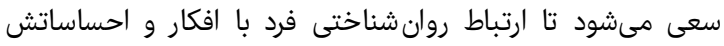

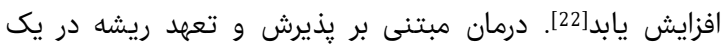

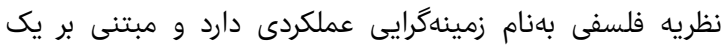

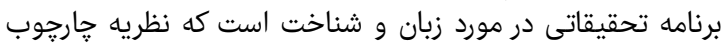

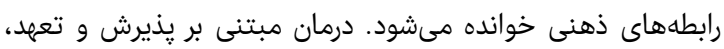

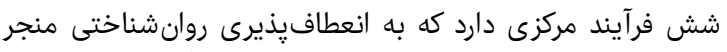

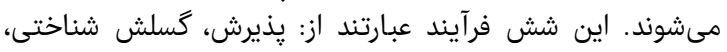

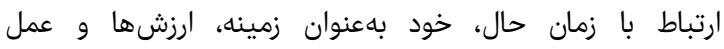

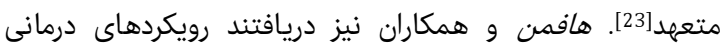

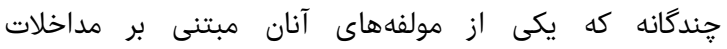

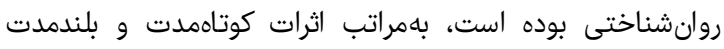

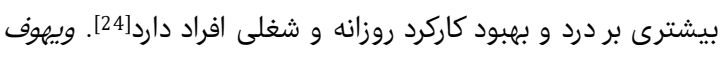

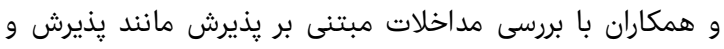

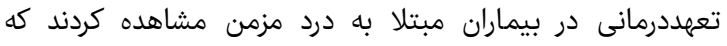

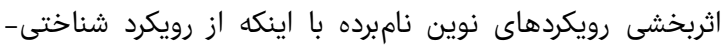

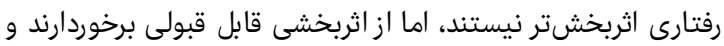

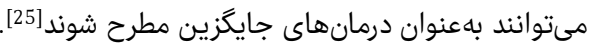

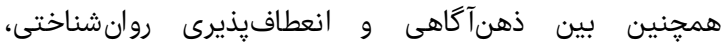

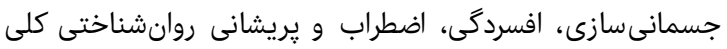

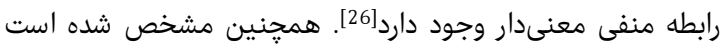

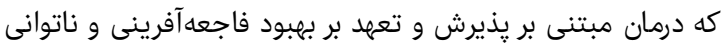
دوره سب، شماره r، بهار 7وسا

\section{درمان مبتنى بر يذيرش و تعهد به ادراك بيمارى و وران

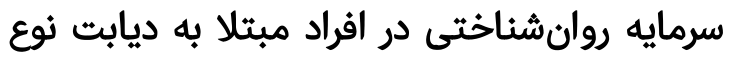

\author{
آسيه باغبان باغستان SA \\ كروه روانشناسى، دانشكده علوم انسانى، دانشكاه بيام نور، تهران، ايران

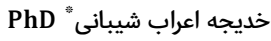

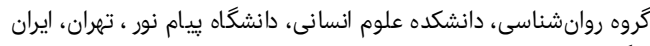

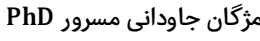

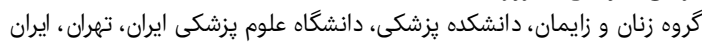

קكيده

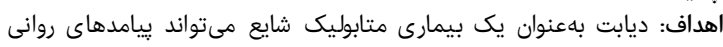

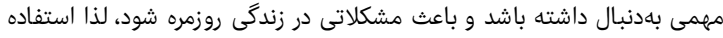

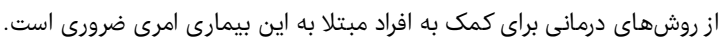

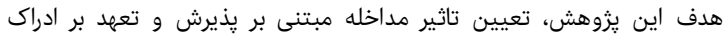

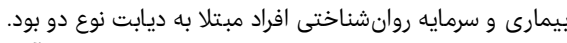

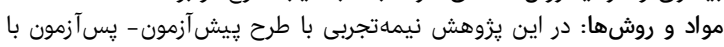

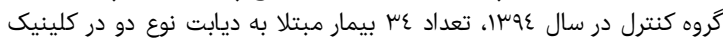

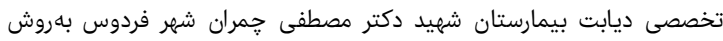

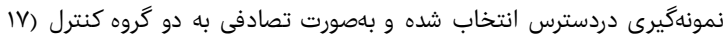

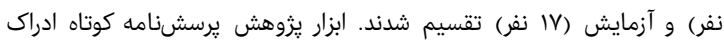

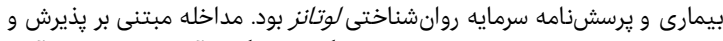

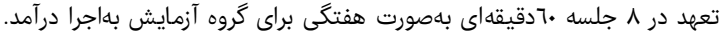

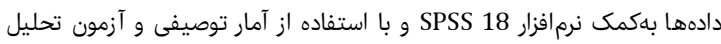

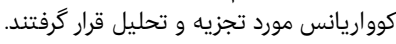

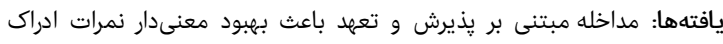

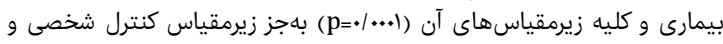

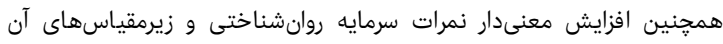
(p=•/...1)

نتيجه گريرى: مداخله مبتنى بر يذيرش و تعهد مى تواند ادراك بيمارى و سرمايه

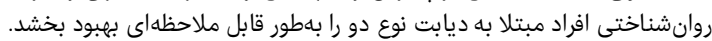

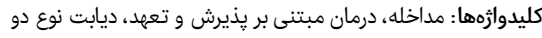

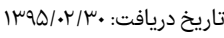

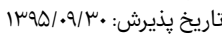

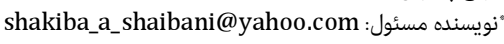

مقدمه

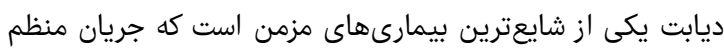

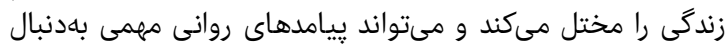

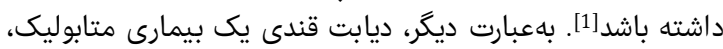

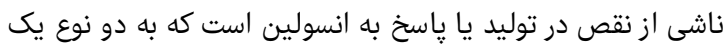

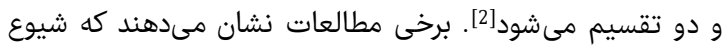

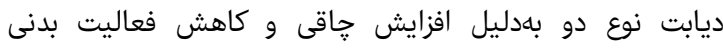

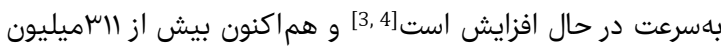

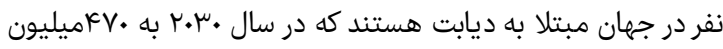

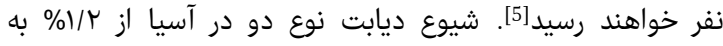

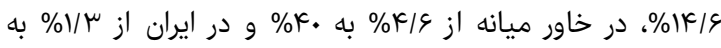

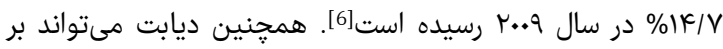

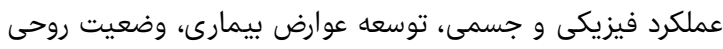

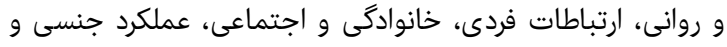

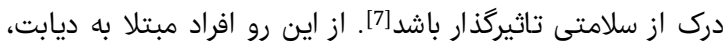

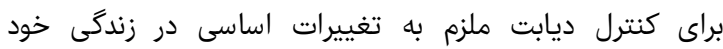

هستند [8].

فصل نامه علمى - يُزوهشى افق دانش 


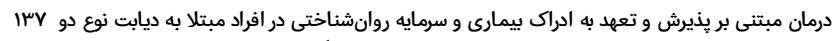

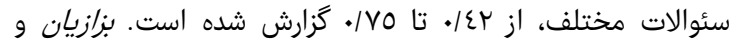

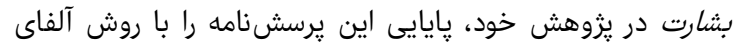

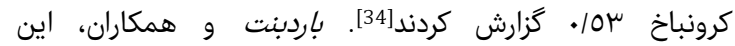

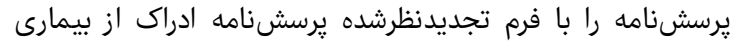

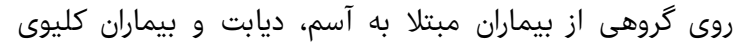

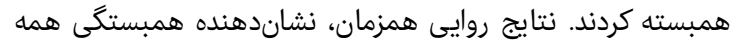

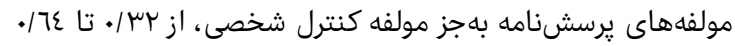

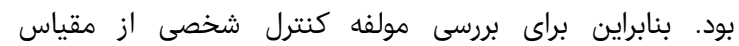

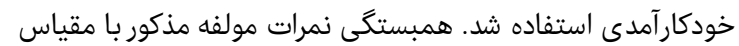

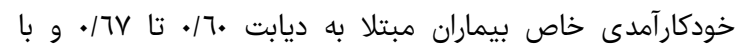

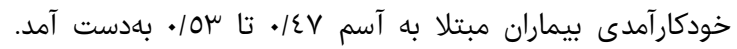

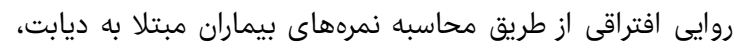

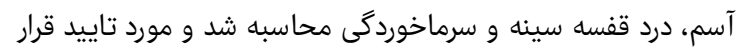

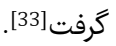

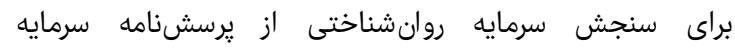

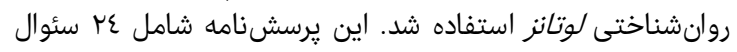

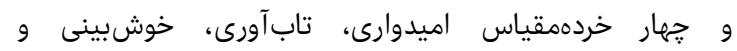
خودكارآمدى است كه هر خردهمقياس شامل 7 گويه است

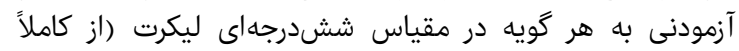

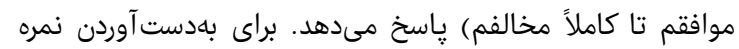

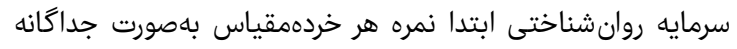

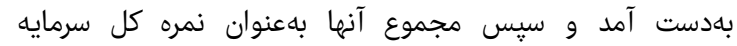

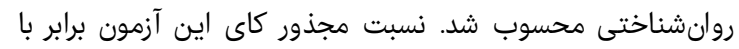

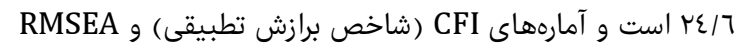

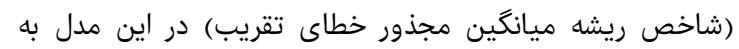

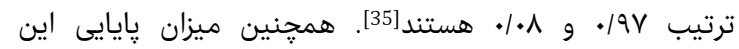

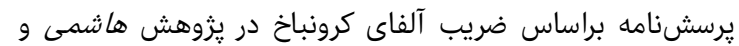
همكاران 110/• بهدست آمدامد [36].

جدول () خلاصه محتواى جلسات آموزشى

\begin{tabular}{|c|}
\hline 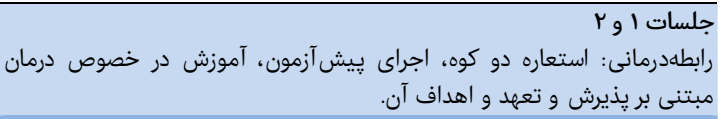 \\
\hline 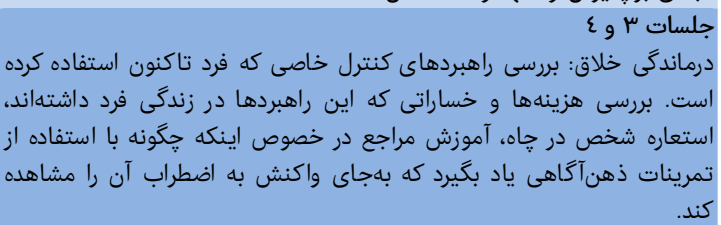 \\
\hline 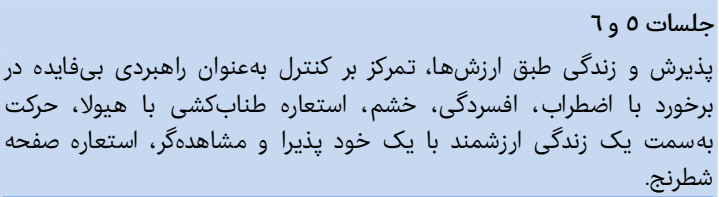 \\
\hline 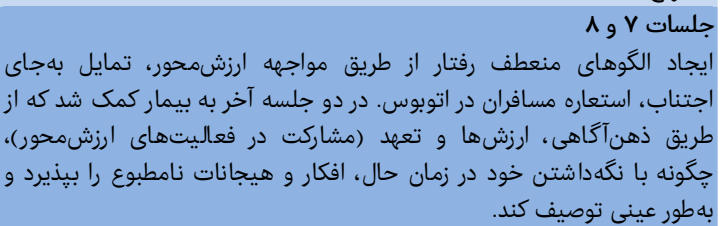 \\
\hline
\end{tabular}

در ابتدا بيماران يس از تماس درمانكر به كلينيك مراجعه كرده و

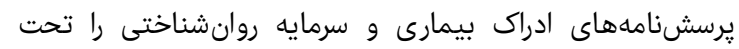

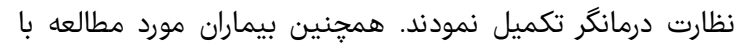

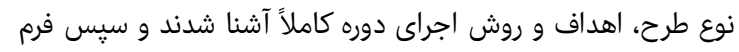

مرتبط با درد، رضايت از زندگى، افسردگى، اضطراب مرتبط با درد،

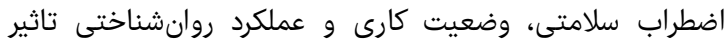

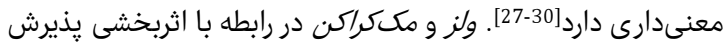

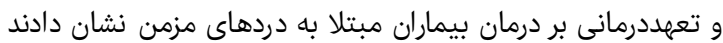

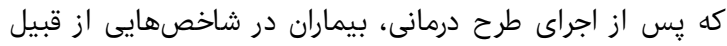

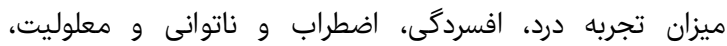

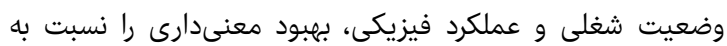

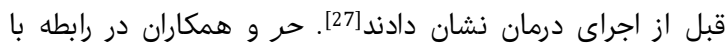

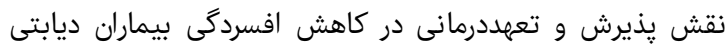

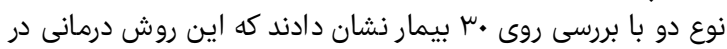

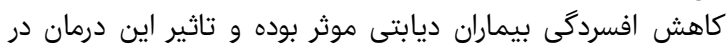

دوره ييكيرى نيز ماندكار بود [31].

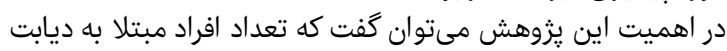

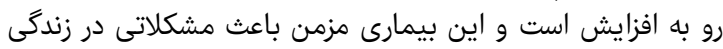

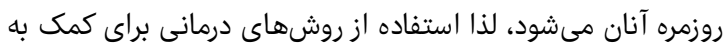

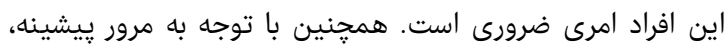

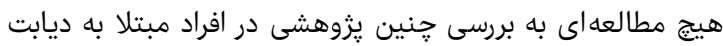

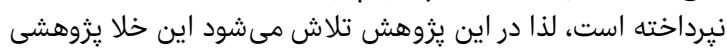

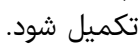

بنابراين هدف اين يزوهش، شود، تعيين تاثير مداخله مبتنى بر يذيرش

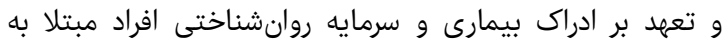

ديابت نوع دو بود.

مواد و روشها

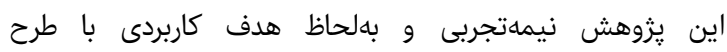

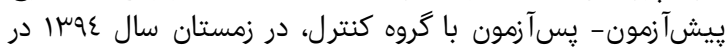

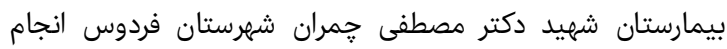

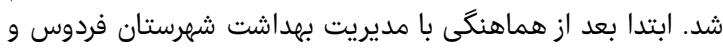

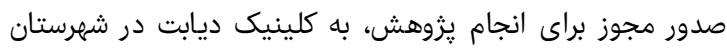

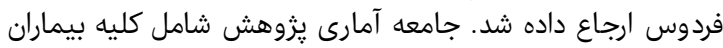

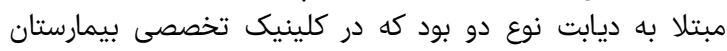

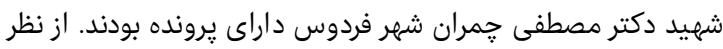

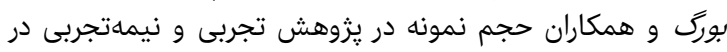

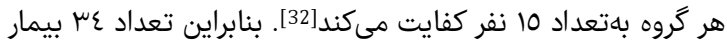

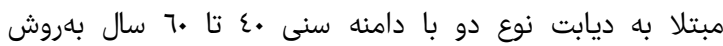

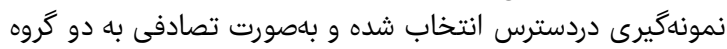

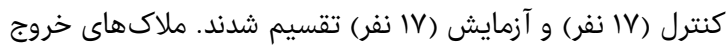

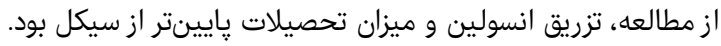

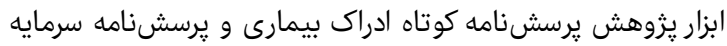

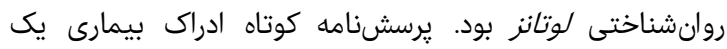

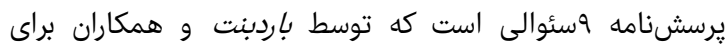

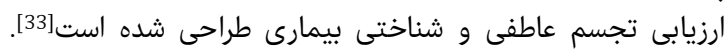

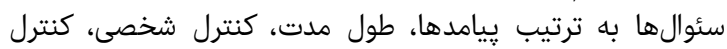

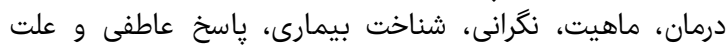

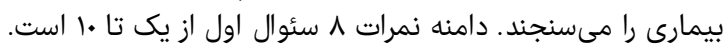

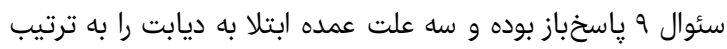

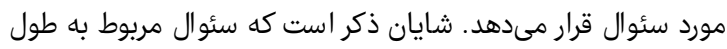

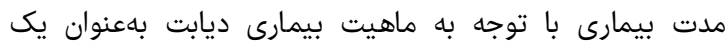

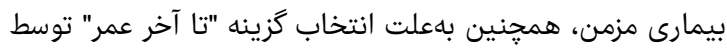

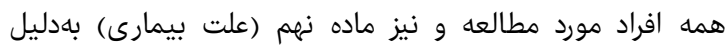

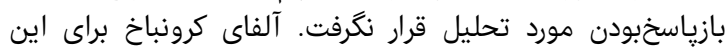

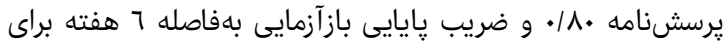




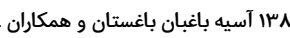

يافتهها

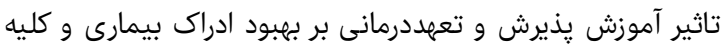

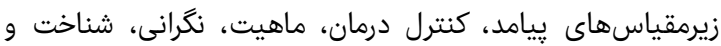

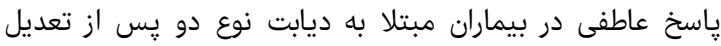

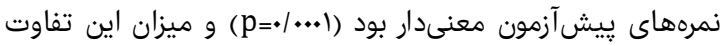

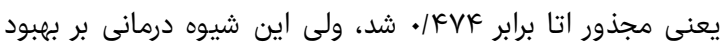

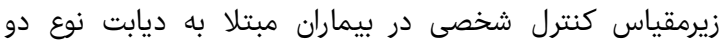

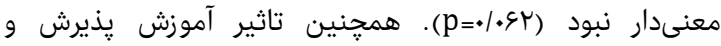

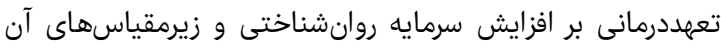

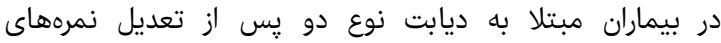

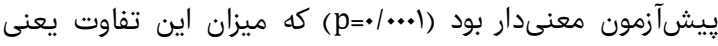

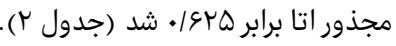

بحث

يثروهش حاضر با هدف بررسى اثربخشى مداخله مبتنى بر يذيرش و

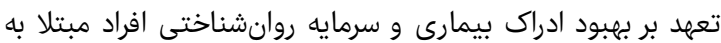

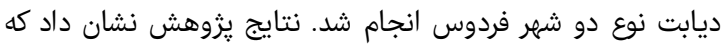

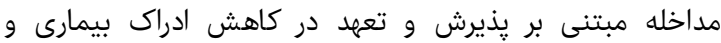

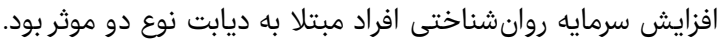

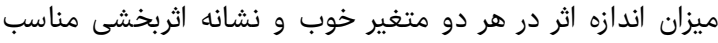

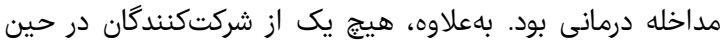

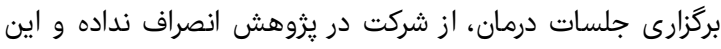

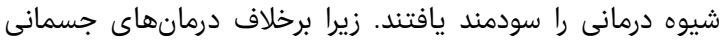

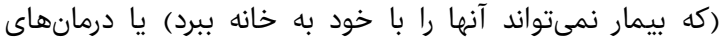

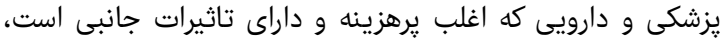

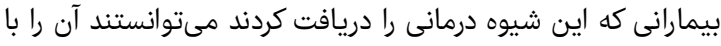

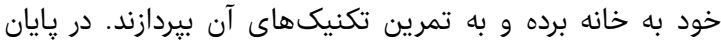

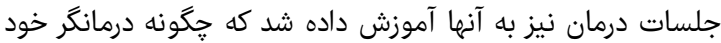

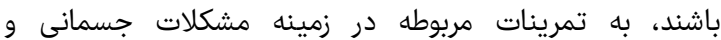

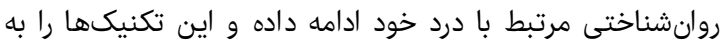

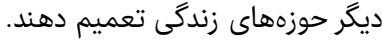

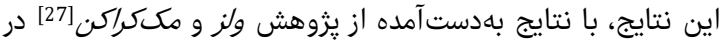

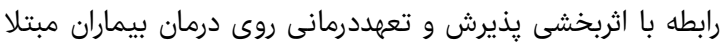

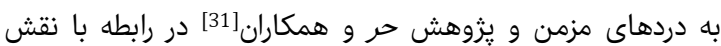

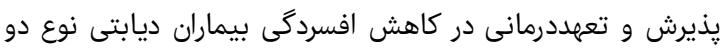

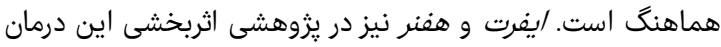

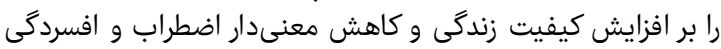

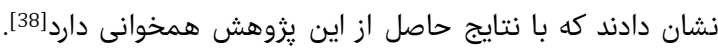

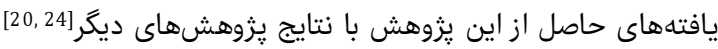

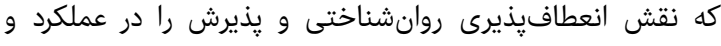

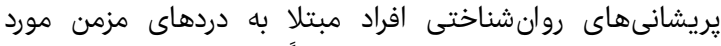

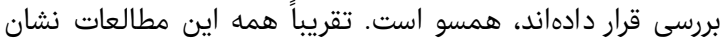

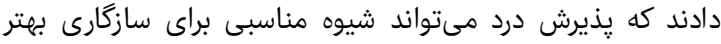

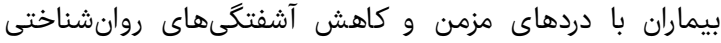

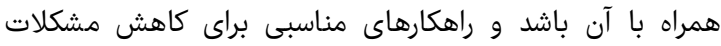
روانشناختى افراد مبتلا به درد مزمن ارئ ارايه كند.

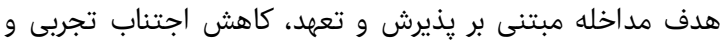

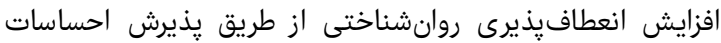

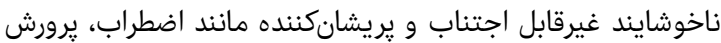

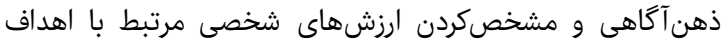

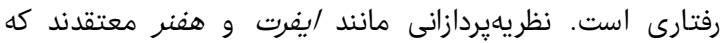

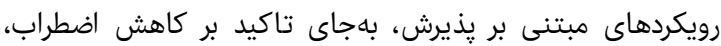

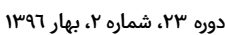

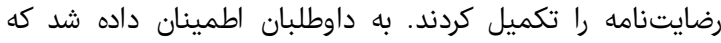

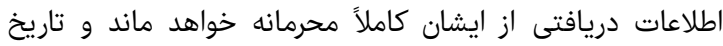
اولين جلسه كلاس اعلام شد.

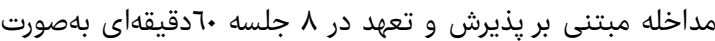

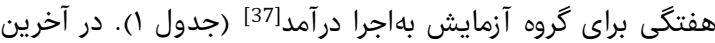

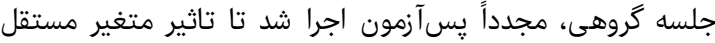

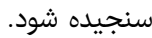

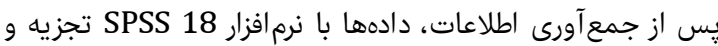

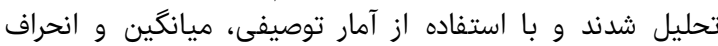

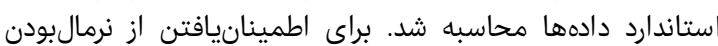

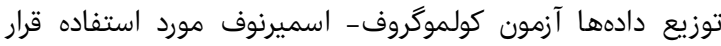

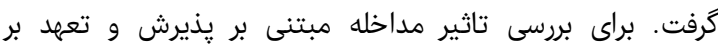

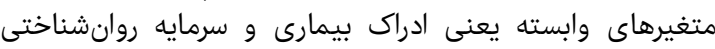

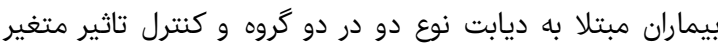
ييش آزمون، از آزمون تحليل كوواريانس استفاده شد.

جدول r) مقايسه ميانگين آمارى نمرات ادراك بيمارى و سرمايه روانشناختى و

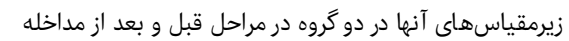

\begin{tabular}{|c|c|c|}
\hline كروه كنترل & كروه آزمايش & متغيرها \\
\hline & & كل ادراك بيمارى \\
\hline$G / 1 \wedge \pm I / F V$ & $\Delta / K V \pm \cdot / \cdot V$ & آزمون \\
\hline$\varepsilon / \mu \Delta \pm 1 / \Delta F$ & $F / V I \pm . / 9 r$ & آزمون \\
\hline & & زيرمقياس ييامد \\
\hline$r / \varepsilon \Delta \pm r / V K$ & $\kappa / V \varphi \pm I / \wedge \Delta$ & أزمون \\
\hline$r / V I \pm Y / 9 V$ & $r / V \varphi \pm 1 / \varepsilon$. & زمون \\
\hline & & زيرمقياس كنترل شخصى \\
\hline$s|4| \pm \mid / \Delta \Lambda$ & $r / V G \pm I / \wedge r$ & أزمون \\
\hline$\varepsilon / \mu \Delta \pm 1 / q \mu$ & $\Delta / \Delta q \pm 1 / \& \mathrm{r}$ & زمون \\
\hline & & زيرمقياس كنترل درمان \\
\hline$G / Y I \pm Y / \cdot r$ & $\Delta / \neg \varphi_{ \pm 1 / \mu}$ & 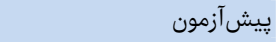 \\
\hline$V / . \varphi \pm r / \varphi \varepsilon$ & $\Lambda / K V \pm I / \& \&$ & يس آزمون \\
\hline & & 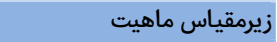 \\
\hline$\varepsilon / r q \pm r / r q \varepsilon$ & $\Delta / r q \pm r / r \mu$ & 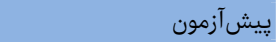 \\
\hline$\varepsilon / \Delta \mu \pm r / q$. & $F / . \xi \pm 1 / F \Lambda$ & يس آزمون \\
\hline & & 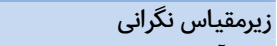 \\
\hline$\varepsilon / \cdot . \pm \mu / 1 \varepsilon$ & $\Delta / \varsigma \Delta \pm r / \Delta r$ & 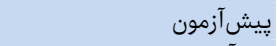 \\
\hline$\varphi / \cdot \varphi \pm \mu / \Lambda$. & $\mu / . \varphi \pm 1 / q \Lambda$ & يس آزمون \\
\hline & & زيرمقياس شناخت بيمارى \\
\hline$q / Y q \pm \mid / V r$ & $\Delta / Q r \pm 1 / \Delta r$ & 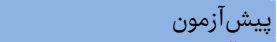 \\
\hline $9 / 4| \pm| / q 1$ & $V / \varepsilon \Delta \pm 1 / \mu^{\mu} V$ & يسآزمون \\
\hline & & ياس پاسخ عاطفى \\
\hline$\Delta / q \kappa \pm r / q$. & $\Delta / \Delta Q \pm r / k r$ & آزمون \\
\hline$\Delta / \& \Delta \pm r / \wedge q$ & $r / \Lambda Y \pm I / \Lambda \Lambda$ & يس آزمون \\
\hline & & نمره كل سرمايه روانشناختى \\
\hline$\psi / \cdot . \pm \cdot|q|$ & $\Psi / \Lambda \Lambda \pm \cdot / \Delta \mu$ & ي تيش آزمون \\
\hline$r / 9 \mu_{ \pm} \cdot / \wedge \mu$ & $K / \Delta \mu_{ \pm} \cdot 1 \& r$ & 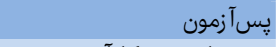 \\
\hline & & باس خودكارآمدى \\
\hline$r / 9 K_{ \pm} \cdot / V Q$ & $m / \cdot \varphi_{ \pm \cdot} / q$. & 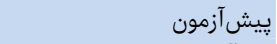 \\
\hline$r / \Lambda \Lambda \pm I / \cdot \Delta$ & $\kappa / \backslash \Lambda \pm \cdot / \wedge \Lambda$ & يس آزمون \\
\hline & & زيرمقياس اميدوارى \\
\hline$H / \Delta H_{ \pm} \cdot|\Delta|$ & $\mu / \Lambda \Lambda \pm \cdot / \varphi$ & 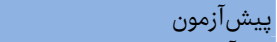 \\
\hline$H / \Delta H_{ \pm} \cdot|\Delta|$ & $\Delta / \mu \omega \pm|c|$ & يسآزمون \\
\hline & & زيرمقياس تابآورى \\
\hline$r / 9 \mu_{ \pm} \cdot / 9$. & $\mu / \mu K_{ \pm} \cdot / \wedge \mu$ & 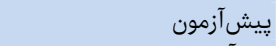 \\
\hline$\mu / / r \pm \cdot / q \mu$ & $F / \& \Delta \pm \cdot / q \mu$ & يسآزمون \\
\hline & & زيرمقياس خوشبينى \\
\hline$r / \wedge \Lambda \pm • / 99$ & $\mu / I r \pm \cdot / \varepsilon$. & 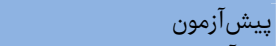 \\
\hline$\mu / . . \pm \cdot / q \mu$ & $k / r q \pm \cdot / V V$ & يسآزمون \\
\hline
\end{tabular}

فصل نامه علمى - يزروهشى افق دانش 
درمان مبتنى بر يذيرش و تعهد به ادراك بيمارى و سرمايه روانشناختى در افراد مبتلا به ديابت نوع دو وسا [Persian]

2- Van Dijk SE, Pols AD, Adriaanse MC, Bosmans JE, Elders PJ, van Marwijk HW, et al. Costeffectivenessof a stepped-care intervention to prevent major depression in patients with type 2 diabetes mellitus and/or coronary heart disease and subthreshold depression: design of a cluster-randomized controlled trial. BMC Psychiatry. 2013;13:128.

3- Azimi H, Yekkeh Fallah L, Ghorbani A. Comparing effects of walking and yoga exercise on quality of life in patients with type 2 diabetes. Prev Care Nurs Midwifery J. 2015;5(1):46-58.

4- Sharma M, Knowlden AP. Role of yoga in preventing and controlling type 2 diabetes mellitus. J Evid Based Complement Altern Med. 2012;17(2):88-95.

5- Azimi-Nezhad M, Ghayour-Mobarhan M, Parizadeh MR, Safarian M, Esmaeili H, Parizadeh SM, et al. Prevalence of type 2 diabetes mellitus in Iran and its relationship with gender, urbanisation, education, marital status and occupation. Singapore Med J. 2008;49(7):571-6.

6- Innes KE, Vincent HK. The influence of yoga-based programs on risk profiles in adults with type 2 diabetes mellitus: A systematic review. Evid Based Complement Alternat Med. 2007;4(4):469-86.

7- Graham JE, Stoebner-May DG, Ostir GV, Snih SA, Peek $\mathrm{MK}$, Markides $\mathrm{K}$, et al. Health related quality of life in older Mexican Americans with diabetes: A crosssectional study. Health Qual Life Outcomes. 2007;5:39.

8- Lee JR, Kim SA, Yoo JW, Kang YK. The present status of diabetes education and the role recognition as a diabetes educator of nurses in Korea. Diabetes Res Clin Pract. 2007;77(Suppl 1):S199-204.

9- Afshar H, Bagherian Sararodi R, Foroozandeh N, Khorramian N, Daghaghzadeh H, Marasy MR, et al. The Relationship between illness perception and symptom severity in patients with Irritable bowel syndrome. J Isfahan Med Sch. 2011:29(137):526-36. [Persian]

10- van Ittersum MW, van Wilgen CP, Hilberdink WK, Groothoff JW, van der Schans CP. Illness perception inpatients with fibromyalgia. Patient Educ Couns. 2009;74(1):53-60.

11- Dennison L, Moss-Morris R, Chalder T. A review of psychological correlates of adjustment in patients with multiple sclerosis. Clin Psychol Rev. 2009;29(2):141-53.

12- Clarke D, Goosen T. The mediating effects of coping strategies in the relationship between automatic negative thoughts and depression in a clinical sample of diabetes patients. Personal Individ Differ. 2009;46(4): 460-4.

13- Scharloo M, Kaptein AA, Weinman J, Bergman W, Vermeer BJ, Roojimans HG. Patient illness perceptions and coping as predictors of functional status in psoriasis: A 1 year follow up. Br J Dermatol. 2000;142(5):899-907.

14- Luthans $\mathrm{F}$. The need for and meaning of positive organizational behavior. J Organ Behav. 2002;23(6):695706.

15- Wood SE, Wood EG. The world of psychology. 2nd edition. USA: Allyn and Bacon; 1996.

16- Erez A, Judge TA. Relationship of core selfevaluations to goal setting, motivation, and performance. J Appl Psychol. 2001;86(6):1270-9.

17- Judge TA, Bono JE. Relationship of core selfevaluations traits -self-esteem, generalized self-efficacy, locus of control, and emotional stability-with job satisfaction and job performance: A meta-analysis. J Appl Psychol. 2001;86(1):80-92.
تمايل به تجربه آن را يرورش مىدهند و قرارگرفتن در معرض اين

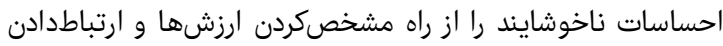

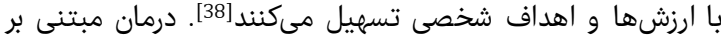

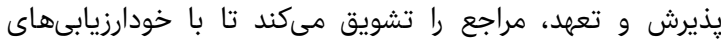

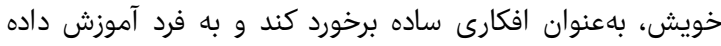

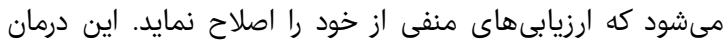

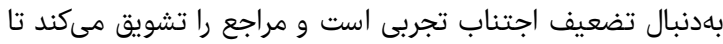

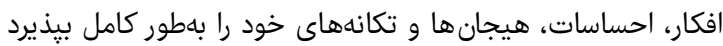

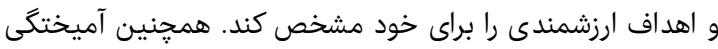

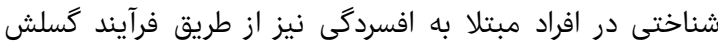

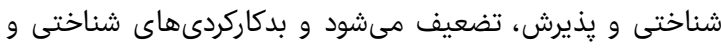

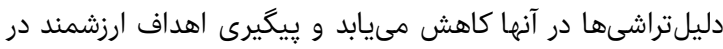

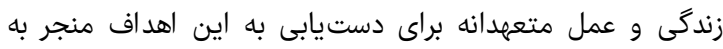

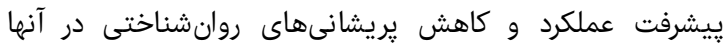
مى شود[39]. با وجود اثربخشبودن مداخله درمانى، اين يزوهش محدوديتهايى

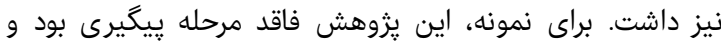

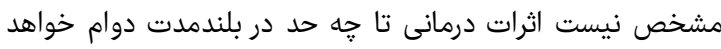

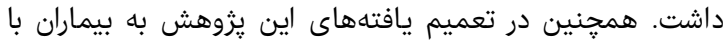

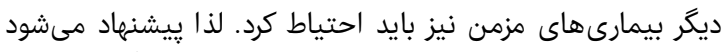

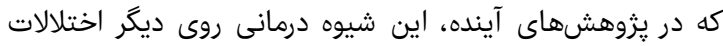

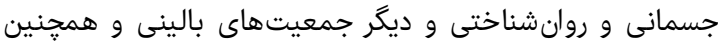

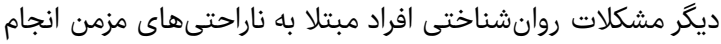

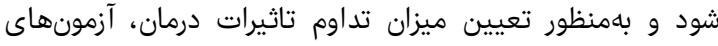

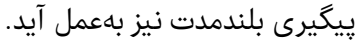

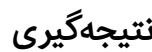

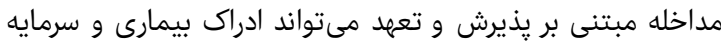

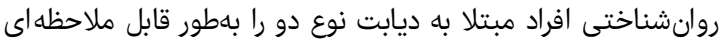

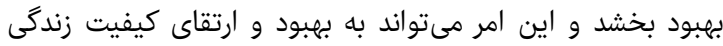
آنها منجر شود.

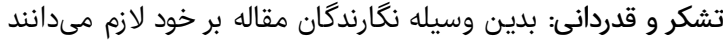

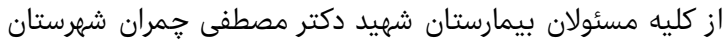

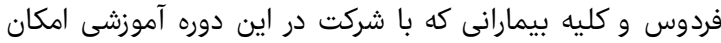

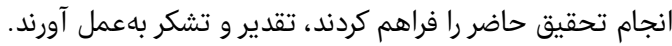

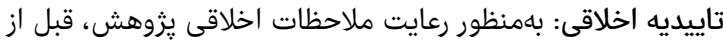

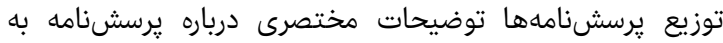

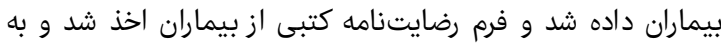

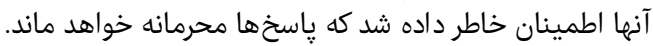

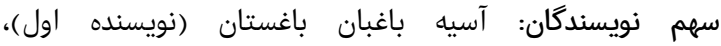
روششناس (•ع\%)؛ خديجه اعراب شيبانى (نويسنده دوم) نكارنده

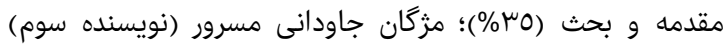

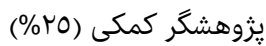
تعارض منافع: هيج گُونه تعارض مناف منافع توسط نويسندگان بيان نشده است. منابع مالى: اين مقاله منبع تامين مالى نداشته است.

منابع 1- Aerab Sheibani K, Akhondi N, Javedani Masrour M, Riahi madvar M. Promotion of psychological capital model in diabetic pation:an explanation of the role of quality of life. J Health Psychol. 2016;5(18):35-51. 
29- Jourdain RL, Dulin PL. "Giving It Space" a case study examining acceptance and commitment therapy for health anxiety in an older male previously exposed to nuclear testing. Clin Case Stud. 2009;8(3):210-25.

30- Johnston M, Foster M, Shennan J, Starkey N, Johnson A. The effectiveness of an acceptance and commitment therapy self-help intervention for chronic pain. Clin J Pain. 2010;26(5):393-402.

31-Hor M, Aghaei A, Abedi A, Attari A. The effectiveness of acceptance and commitment therapy on depression in patients with type 2 diabetes. J Res Behav Sci 2013;11(2):121-8. [Persian]

32- Borg W, Gall M, Gall J. Quantitative and qualitative research methods in Psychology. Nasr AR, Areyzi HR, Abolqasemi M, Pakseresht MJ, Kiamanesh AR, Bagheri Kh, et al, translators. Tehran: Samt Publication; 2015.

33- Bardbent E, Petrie KJ, Main J, Weinman J. The brief illness perception questionnaire. J Psychosom Res. 2006;60(6):631-7.

34- Bazzazian S, Besharat MA, Bahrami Ehsan H, Rajab A. The Moderating Role of Coping Strategies in Relationship Between Illness Perception, Quality of Life and HbA1c in Patients with Type I Diabetes. Iran J Endocrinol Metab. 2010;12(3):213-21. [Persian]

35- Luthans F, Avolio B, Avey J, Norman S. Positive psychological capital: Measurement and relationship with performance and satisfaction. Person Psychol. 2007;60(3):138-46.

36- Hashemi Nosratabad T, Babapour Khairuddin J, Bahadari Khosroshahi J. Role of psychological capital in psychological wellbeing by considering the moderating effects of social capital. Soc Psychol Res. 2012;1(4):12344. [Persian]

37- Abedi MR, Izadi R. Acceptance and commitment therapy. $4^{\text {th }}$ edition. Tehran: Jangal Press; 2015. [Persian] 38- Eifert GH, Heffner M. The effects of acceptance versus control contexts on avoidance of panic-related symptoms. J Behav Ther Exp Psychiatry. 2003;34(34):293-312.

39- Hayes SC, Masuda A, Bissett RT, Luoma J, Guerrero LF. DBT, FAP and ACT: How empirically oriented are the new behavior therapy technologies?. Behav Ther. 2004;35(1):35-54.
18- Parker CP, Baltes BB, Young SA, Huff JW, Altmann RA Lacost HA, et al. Relationships between psychological climate perceptions and work outcomes: A meta-analytic review. J Organ Behav. 2003;24(4):389-416.

19- Creed PA, Machin M, Hicks R. Improving mental health status and coping abilities for long-term unemployed youth using cognitive-behavior therapy based training interventions. J Organ Behav. 1999;20(6):963-78.

20- Robbins S, Waters-Marsh T, Caccioppe R, Millet B. Organisational behaviour. Australia: Pretince Hall; 1994.

21- Killgore WD, Kahn-Greene ET, Lipizzi EL, Newman RA, Kamimori GH, Balkin TJ. Sleep deprivation reduces perceived emotional intelligence and constructive thinking skills. Sleep Med. 2008;9(5):517-26.

22- Rajabi S, Yazdkhasti F. The effectiveness of acceptance and commitment group therapy on anxiety and depression in women with MS who were referred to the MS Association. J Clin Psychol. 2014;6(1):29-39. [Persian]

23- Hayes SC, Luoma JB, Bond FW, Frank WB, Masuda A, Lillis J. Acceptance and commitment therapy: Model, processes and outcomes. Behav Res Ther. 2006;44(1):125.

24- Hoffman BM, Papas RK, Chatkoff DK, Kerns RD. Metaanalysis of psychological interventions for chronic low back pain. Health Psychol. 2007;26(1):1-9.

25- Uman LS. Systematic review and meta-analysis. J Can Acad Child Adolesc Psychiatry. 2011;20(1):57-9.

26- Masuda A, Tully EC. The role of mindfulness and psychological flexibility insomatization, depression, anxiety, and general psychological distress in a nonclinical college sample. J Evid Based Complement Altern Med. 2012;17(1):66-71.

27- Vowles KE, McCracken LM. Acceptance and valuesbased action in chronic pain: A study of treatment effectiveness and process. J Consul Clin Psychol. 2008;76(3):397-407.

28- Wicksell RK, Ahlqvist J, Bring A, Melin L, Olsson GL. Can exposure strategies improve functioning and life satisfaction in people with chronic pain and whiplashassociated disorders (WAD0? a randomized controlled trial. Cogn Behav Ther. 2008;37(3):169-82. 